\title{
A Case of Paracentral Acute Middle Maculopathy Occurring After Pars Plana Vitrectomy
}

\author{
(D) Gulsah Gumus, ${ }^{1}$ (1) Nese Alagoz, ${ }^{2}$ (1) Cengiz Alagoz, ${ }^{2}$ (1) Sehnaz Ozcaliskan, ${ }^{2}$ (1) Ozgur Artunay ${ }^{2}$ \\ ${ }^{1}$ Department of Ophtalmology, Gaziantep Dr. Ersin Arslan Training and Research Hospital, Gaziantep, Turkey \\ ${ }^{2}$ Department of Ophtalmology, Health Sciences University, Istanbul Beyoglu Eye Training and Research Hospital, Istanbul, Turkey
}

\begin{abstract}
Paracentral acute middle maculopathy (PAMM) is a clinical finding that is thought to be a result of ischemia in the middle and deep capillary plexus of the retina. It has been reported in the literature that PAMM may be associated with systemic and vascular risk factors. This report describes a case of PAMM that occurred following a pars plana vitrectomy.

Keywords: Ischemia, paracentral acute middle maculopathy, pars plana vitrectomy
\end{abstract}

\section{Introduction}

Paracentral acute middle maculopathy (PAMM) is a spectraldomain optical coherence tomography (SD-OCT) finding characterized by a hyper-reflective band in the inner nuclear layer (INL). Ischemia in the intermediate (ICP), and deep capillary plexus (DCP) of the retina is thought to be a pathogenic factor (I).

PAMM may be idiopathic or occur in association with retinal vascular pathologies, such as diabetic retinopathy, hypertensive retinopathy, sickle cell retinopathy, retinal vein occlusion, retinal artery occlusion, or retinal vasculitis (I). Cases of PAMM have also been reported in association with migraine, hypovolemia, orbital compression injury, viral disease, or vaccination, and after the use of medications, such as amphetamines, vasopressors, oral contraceptives, or consumption of caffeine $(I, 2)$. This case report is a description of a case of PAMM occurring following a pars plana vitrectomy (PPV) procedure.

\section{Case Report}

A 76-year-old male patient was admitted to the retina clinic with the complaints of visual impairment and metamorphopsia. A detailed medical history included diabetes mellitus (DM) and bypass surgery 6 years prior. An ophthalmological examination yielded a best corrected visual acuity (BCVA) of $20 / 20$ in the right eye and 20/200 in the left eye. No pathology was noted on biomicroscopic examination. The intraocular pressure was $17 \mathrm{~mm} \mathrm{Hg}$ in both eyes. A dilated fundus examination revealed an epiretinal membrane (ERM) and vitreomacular traction (VMT) in the left eye and no pathology of the retina in the right eye. SD-OCT images confirmed the existence of VMT concurrent with an ERM (Fig. Ia). PPV and ERM and internal limiting membrane (ILM) peeling was performed in the left eye. Air was used as an endotamponade at the conclusion of the surgery.

The patient's BCVA was 20/800 and he described a paracentral scotoma in the left eye I week after the PPV.

How to cite this article: Gumus G, Alagoz N, Alagoz C, Ozcaliskan S, Artunay O. A Case of Paracentral Acute Middle Maculopathy Occurring After Pars Plana Vitrectomy. Beyoglu Eye J 2021; 6(2): I55-157.

Address for correspondence: Gulsah Gumus, MD. Gaziantep Dr. Ersin Arslan Egitim ve Arastirma Hastanesi, Gaziantep, Turkey Phone: +90 5535785988 E-mail: gumus_118_@hotmail.com

Submitted Date: January 12, 2021 Accepted Date: February 10, 2021 Available Online Date: June 08, 2021

${ }^{\circ}$ Copyright 2021 by Beyoglu Eye Training and Research Hospital - Available online at www.beyoglueye.com OPEN ACCESS This work is licensed under a Creative Commons Attribution-NonCommercial 4.0 International License. 
A fundus examination revealed a gray-white lesion located deep in the paracentral fovea of the left eye. Near-infrared imaging revealed a gray-white lesion corresponding to the same area. A hyper-reflective, band-like lesion in the INL was observed on macular SD-OCT (Fig. Ib). The patient was diagnosed with PAMM following PPV and followed up for 6 months. The BCVA in the left eye gradually improved to $20 / 50$. Macular SD-OCT imaging illustrated marked atrophy of the middle retinal layers of the corresponding region (Fig. Ic).

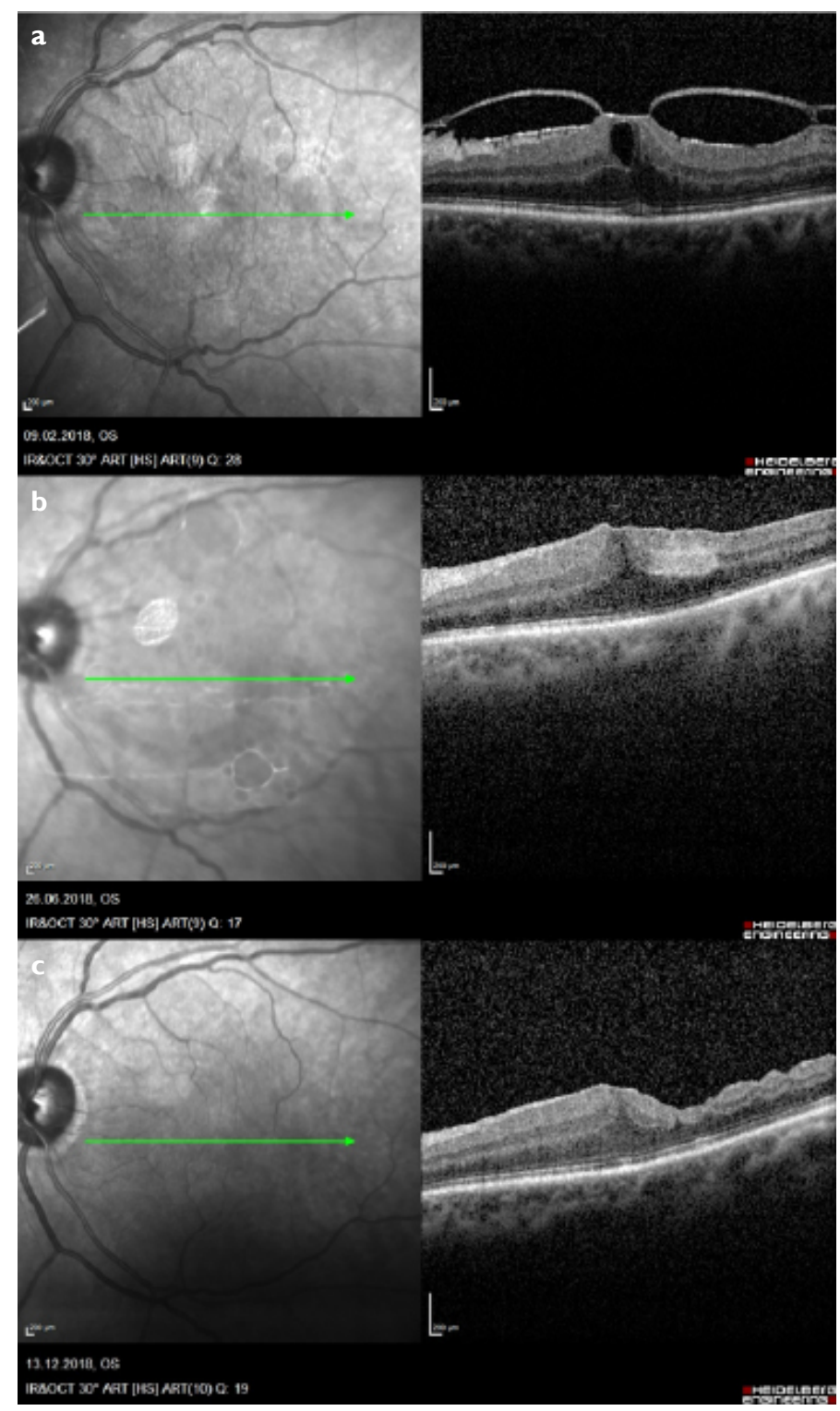

Figure I. (a) Preoperative spectral-domain optical coherence tomography (SD-OCT) images illustrating an epiretinal membrane, (b) postoperative I week SD-OCT images demonstrating a parafoveal hyper-reflective band with distinct margins in the inner nuclear layer, (c) SD-OCT images recorded at postoperative 6 months revealing retinal atrophy in the inner layers in the temporal region of the macula.

\section{Discussion}

Sarraf et al. first described a PAMM lesion as characterized by a hyper-reflective band in the INL in the acute phase visible on SD-OCT images that progresses to atrophy of the INL in the chronic phase (I).

The retinal capillary system feeds the neurosensory retina via 3 plexuses: the superficial (SCP), the ICP, and the DCP. The SCP is located within the ganglion cell layer, while the ICP and the DCP are located at the inner and outer borders of the INL. Fundus fluorescein angiography (FA) can only illustrate the SCP; it is not capable of demonstrating occlusion in the ICP or the DCP $(I, 3)$. SCP occlusions are associated with soft exudates, while ICP and DCP occlusions appear as a deep, gray-white lesion with a more prominent edges (3).

The presence of a hyper-reflective band in the INL visible with SD-OCT suggests that a PAMM lesion may have developed as the result of ICP and/or DCP ischemia. OCT angiography (OCT-A) imaging may indicate that a lesion was the result of capillary drop-out (I) or demonstrate occlusion in the ICP and/or DCP, which may also be accompanied by an SCP obstruction (4).

In the present case, we observed a gray-white, deeply located lesion in the paracentral fovea occurring following a PPV procedure. SD-OCT showed a hyper-reflective, bandlike lesion in the INL of the same region. The vascular origin of the lesion points to the probability of the coexistence of other systemic and retinal vascular pathologies. Our patient had systemic vascular risk factors of DM and coronary arterial disease.

Retinal arterial occlusions are a rare occurrence after intraocular surgery; however, increased intraocular pressure and the use of subtenon, peribulbar, or retrobulbar anesthesia during ocular surgery may disrupt ocular perfusion and cause vascular occlusion (5). In this case, the patient developed a PAMM lesion following a PPV.

There is no current treatment for PAMM. Diagnosis and treatment of systemic and vascular risk factors should be addressed accordingly. Persistent scotoma, attenuation, and atrophy may develop in the INL and OPL (I). Full resolution of scotomas has been reported but partial resolution is the most frequent outcome. Significant visual impairment is uncommon.

\section{Disclosures}

Informed consent: Written informed consent was obtained from the patient for the publication of the case report and the accompanying images.

Peer-review: Externally peer-reviewed.

Conflict of Interest: None declared.

Authorship Contributions: Involved in design and conduct of the study (GG, NA, CA); preparation and review of the study (SO, $O A)$; data collection (GG, CA). 


\section{References}

I. Rahimy E, Kuehlewein L, Sadda SR, Sarraf D. Paracentral acute middle maculopathy: what we knew then and what we know now. Retina 2015;35:1921-30. [CrossRef]

2. Chen X, Rahimy E, Sergott RC, Nunes RP, Souza EC, Choudhry $\mathrm{N}$, et al. Spectrum of retinal vascular diseases associated with paracentral acute middle maculopathy. Am J Ophthalmol 2015;160:26-34.el. [CrossRef]

3. Yu S, Pang CE, Gong Y, Freund KB, Yannuzzi LA, Rahimy E, et al.
The spectrum of superficial and deep capillary ischemia in retinal artery occlusion. Am J Ophthalmol 20I5; I59:53-63.el-2

4. Chu S, Nesper PL, Soetikno BT, Bakri SJ, Fawzi AA. Projectionresolved OCT angiography of microvascular changes in paracentral acute middle maculopathy and acute macular neuroretinopathy. Invest Ophthalmol Vis Sci 2018;59:29I3-22. [CrossRef]

5. Fischer C, Bruggemann A, Hager A, Callizo Planas J, Roider J, Hoerauf $\mathrm{H}$. Vascular occlusions following ocular surgical procedures: A clinical observation of vascular complications after ocular surgery. J Ophthalmol 2017;20I7:9120892. [CrossRef] 